\title{
Radio Emission from Supernovae
}

\author{
Kurt W. Weiler*, Nino Panagia ${ }^{\dagger, *}$, Richard A. Sramek ${ }^{\S}$, Schuyler D. Van \\ Dyk $^{\|}$, Christopher L. Williams ${ }^{*}$, , Christopher J. Stockdale ${ }^{\dagger \dagger}$ and Matthew \\ T. Kelley ${ }^{\dagger \dagger}$ \\ ${ }^{*}$ Naval Research Laboratory, Code 7210, Washington, DC 20375-5351, USA \\ ${ }^{\dagger}$ Space Telescope Science Institute, 3700 San Martin Drive, Baltimore, MD 21218, USA \\ ${ }^{* *}$ INAF/Osservatorio Astrofisico di Catania, Via S.Sofia 78, I-95123 Catania, Italy \\ ${ }^{\ddagger}$ Supernova Ltd., Olde Yard Village \#131, Northsound Road, Virgin Gorda, British Virgin Islands \\ ${ }^{\S}$ PO Box 0, National Radio Astronomy Observatory, Socorro, NM 87801, USA \\ IIPAC/Caltech, Mail Code 100-22, Pasadena, CA 91125, USA \\ "Present address: Massachusetts Institute of Technology, Kavli Institute for Astrophysics and \\ Space Research, Cambridge, MA 02139 \\ ${ }^{\dagger}$ Marquette University, Physics Department, P.O. Box 1881, Milwaukee, WI 53214-1881, USA
}

\begin{abstract}
Study of radio supernovae over the past 27 years includes more than three dozen detected objects and more than 150 upper limits. From this work it is possible to identify classes of radio properties, demonstrate conformance to and deviations from existing models, estimate the density and structure of the circumstellar material and, by inference, the evolution of the presupernova stellar wind, and reveal the last stages of stellar evolution before explosion. It is also possible to detect ionized hydrogen along the line of sight, to demonstrate binary properties of the presupernova stellar system, and to detect clumpiness of the circumstellar material.

Along with reviewing these general properties of the radio emission from supernovae, we present our extensive observations of the radio emission from supernova (SN) 1993J in M 81 (NGC 3031) made with the Very Large Array and other radio telescopes. The SN 1993J radio emission evolves regularly in both time and frequency, and the usual interpretation in terms of shock interaction with a circumstellar medium (CSM) formed by a pre-supernova stellar wind describes the observations rather well considering the complexity of the phenomenon. However: 1) The highest frequency measurements at $85-110 \mathrm{GHz}$ at early times ( $<40$ days) are not well fitted by the parameterization which describes the $\mathrm{cm}$ wavelength measurements rather well. 2) At mid-cm wavelengths there is often deviation from the fitted radio light curves, particularly near the peak flux density, and considerable shorter term deviations in the declining portion when the emission has become optically thin. 3 ) At a time $\sim 3100$ days after shock breakout, the decline rate of the radio emission steepens from $\left(\mathrm{t}^{+\beta}\right) \beta \sim-0.7$ to $\beta \sim-2.7$ without change in the spectral index $\left(v^{+\alpha} ; \alpha \sim-0.81\right)$. However, this decline is best described not as a power-law, but as an exponential decay starting at day $\sim 3100$ with an e-folding time of $\sim 1100$ days. 4) The best overall fit to all of the data is a model including both non-thermal synchrotron self-absorption (SSA) and a thermal free-free absorbing (FFA) components at early times, evolving to a constant spectral index, optically thin decline rate, until a break in that decline rate at day $\sim 3100$, as mentioned above. Moreover, neither a purely SSA nor a purely FFA absorbing model can provide a fit that simultaneously reproduces the light curves, the spectral index evolution, and the brightness temperature evolution.
\end{abstract}

Keywords: radio continuum:stars - supernovae

PACS: $97.60 . \mathrm{Bw}, 98.58 . \mathrm{Mj}$

CP937, Supernova 1987A: 20 Years After, edited by S. Immler, K. Weiler, and R. McCray (C) 2007 American Institute of Physics 978-0-7354-0448-9/07/\$23.00 


\section{INTRODUCTION}

A series of papers on radio supernovae (RSNe) has established the radio detection and, in a number of cases, radio evolution for more than three dozen supernovae (SNe). A much larger list of more than 150 additional SNe have low radio upper limits (See $h t t p: / / r s d$ www.nrl.navy.mil/7213/weiler/kwdata/rsnhead.html). In this extensive study of the radio emission from $\mathrm{SNe}$, several effects have been noted:

- type Ia SNe are not radio emitters to the detection limit of the VLA ${ }^{1}$

- type $\mathrm{Ib} / \mathrm{c} \mathrm{SNe}$ are radio luminous with steep spectral indices (generally $\alpha<-1$; $S \propto v^{+\alpha}$ ) and have a fast turn-on/turn-off, usually peaking at $6 \mathrm{~cm}$ near or before optical maximum.

- type II SNe show a range of radio luminosities with flatter spectral indices (generally $\alpha>-1$ ) and a relatively slow turn-on/turn-off, usually peaking at $6 \mathrm{~cm}$ significantly after optical maximum.

Measurements of the multi-frequency radio light curves and their evolution with time show the density and structure of the CSM, evidence for possible binary companions, clumpiness or filamentation in the presupernova wind, mass-loss rates and changes therein for the presupernova stellar system and, through stellar evolution models, estimates of the ZAMS presupernova stellar mass and the stages through which the star passed on its way to explosion.

Of particular recent interest are the radio observations of SN 1993J in M 81 (NGC 3031) which was discovered at magnitude $V \sim 11^{\mathrm{m}} 8$ on 28.91 March 1993 [28], and by 30 March, at maximum optical magnitude $V=10^{\mathrm{m}} 7$, had become the brightest supernova in the northern hemisphere since SN 1954A. Hydrogen was soon identified in its optical spectrum, classifying it as a type II SN (SNII; see, e.g., [1, 10]).

From the outset, SN 1993J displayed unusual characteristics for a SNII. The visual light curve was markedly different from both the SNIIL (linear) and SNIIP (plateau) subtypes in that it exhibited a second maximum $\sim 17$ days after the first one. Its unusual light curve and spectrum were quickly interpreted by Nomoto et al. [21] and Schwartz et al. [30] as implying a red supergiant progenitor with a thin hydrogen envelope which would spectrally evolve from resembling a SNII to resembling a SNIb, thereby suggesting a SNIIb classification. Continuing observations of visual spectra by Filippenko et al. [11] confirmed this transition.

Radio emission from SN 1993J was detected with the VLA on UT 02.30 April 1993, with a flux density of $0.8 \pm 0.2 \mathrm{mJy}$ at $1.3 \mathrm{~cm}$ ([39], see also [32,33]) and with the Ryle Telescope in Cambridge, UK on UT 5.7 April 1993 at $2 \mathrm{~cm}$ [24]. By UT 25 April 1993 the rapidly expanding SN already had a measurable size of $0.25 \pm 0.1$ milliarcseconds (mas) with Very Long Baseline Interferometry (VLBI) techniques [13, 14].

High angular resolution VLBI size measurements of the expanding SN were conducted very early by Marcaide et al. [15] and Bartel et al. [2] and VLBI monitoring

\footnotetext{
1 The VLA telescope of the National Radio Astronomy Observatory is operated by Associated Universi-
} ties, Inc. under a cooperative agreement with the National Science Foundation. 

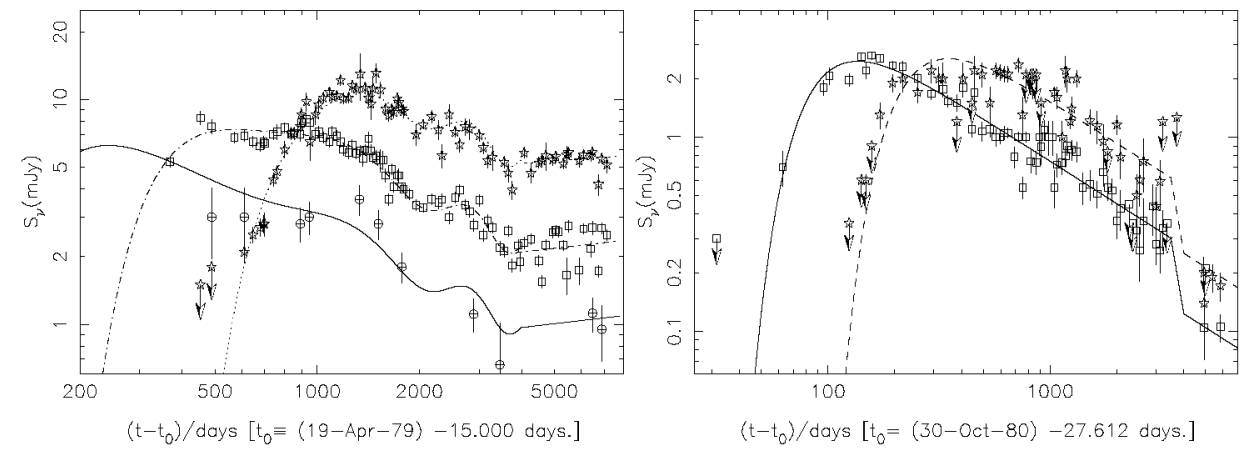

FIGURE 1. Left (1a): Type II SN $1979 \mathrm{C}$ at $2 \mathrm{~cm}(14.9 \mathrm{GHz}$; crossed circles, solid line), $6 \mathrm{~cm}(4.9 \mathrm{GHz}$; open squares, dash-dot line), and $20 \mathrm{~cm}(1.5 \mathrm{GHz}$; open stars, dotted line) (Note that the radio flux density increases after day $\sim 4000$ and has a sinusoidal modulation before day $\sim 4000 ;[20,36,37]$.) Right $(1 \mathrm{~b})$ : Type II SN 1980K at $6 \mathrm{~cm}(4.9 \mathrm{GHz}$; open squares, solid line), and $20 \mathrm{~cm}(1.5 \mathrm{GHz}$; open stars, dashed line). (Note a sharp drop in flux density after day $\sim 4000 ;[19,38]$ )

continues to the present at multiple wavelengths (see, e.g., $[3,4,5,16,17]$ ).

Extensive radio monitoring of the integrated flux density of SN 1993J has been conducted by the VLA [34, 42] and with the Ryle Telescope [25]. Very short wavelength observations were conducted with the French-German Institut de Radioastronomie Millimetrique (IRAM) telescope [26] and at the Caltech Owens Valley Radio Observatory (OVRO) [22, 23]. More recently, Chandra et al. [6] have conducted longer wavelength observations with the Giant Metrewave Radio Telescope (GMRT).

\section{EMISSION MODELS}

All known RSNe appear to share common properties of:

- Nonthermal synchrotron emission with high brightness temperature.

- A decrease in absorption with time, resulting in a smooth, rapid turn-on first at shorter wavelengths and later at longer wavelengths.

- A power-law decline of the flux density with time at each wavelength after maximum flux density (optical depth $\sim 1$ ) is reached at that wavelength.

The characteristic RSN radio light curves arise from the competing effects of slowly declining non-thermal radio emission and more rapidly declining thermal and/or nonthermal absorption yielding a rapid turn-on and slower turn-off of the radio emission at any single frequency. This characteristic light curve shape is illustrated in Figure 1 for SN 1979C and SN 1980K. Since absorption processes are greater at lower frequencies, transition from optically thick to optically thin (turn-on) occurs first at higher frequencies and later at lower frequencies.

Chevalier $[7,8]$ has proposed that the relativistic electrons and enhanced magnetic field necessary for synchrotron emission arise from the SN blastwave interacting with a relatively high density CSM which has been ionized and heated by the initial UV/X-ray 
flash. This CSM density $(\rho)$, which decreases as an inverse power, $s$, of the radius, $r$, from the star, is presumed to have been established by a presupernova stellar wind with mass-

loss rate, $\dot{M}$, and velocity, $w_{\text {wind }}$, (i.e., $\rho \propto \frac{\dot{M}}{w_{\text {wind }} r^{s}}$ ) from a massive stellar progenitor or companion. For a constant mass-loss rate and constant wind velocity $s=2$. This ionized CSM is the source of some or all of the initial thermal gas free-free absorption (FFA). Additionally, Chevalier [9] has proposed that synchrotron self-absorption (SSA) may play a role in some objects.

A rapid rise in the observed radio flux density results from a decrease in these absorption processes as the radio emitting region expands and the absorption processes, either internal or along the line-of-sight, decrease. Weiler et al. [35] have suggested that this CSM can be "clumpy" or "filamentary," leading to a slower radio turn-on, and Montes et al. [18] have proposed the possible presence of a distant ionized medium along the line-of-sight which is sufficiently distant from the explosion that it is unaffected by the blastwave and can cause a spectral turn-over at low radio frequencies. In addition to clumps or filaments, the CSM may be radially structured with significant density irregularities such as rings, disks, shells, or gradients.

\section{RESULTS}

The success of the basic parameterization and modeling has been shown in the good correspondence between the model fits and the data for all subtypes of RSNe: e.g., type Ib SN 1983N [31], type Ic SN 1990B [32], and type II SN 1979C [20, 36, 37] and SN $1980 \mathrm{~K}[19,38]$. (Note that after day $\sim 4000$, the evolution of the radio emission from both SN 1979C and SN 1980K deviates from the expected model evolution and that SN 1979C shows a sinusoidal modulation in its flux density prior to day $\sim 4000$.)

Additionally, in their study of the radio emission from SN 1986J, Weiler et al. [35] found that the simple Chevalier model could not describe the relatively slow turn-on. They therefore included additional terms in their model (see, e.g., [40, 41]) which they interpreted [35] to represent the presence of filaments or clumps in the CSM. Such a clumpiness in the wind material was again required for modeling the radio data from SN 1988Z [33, 43] and SN 1993J [34]. Since that time, evidence for filamentation in the envelopes of SNe has also been found from optical and UV observations [12, 29].

Although there is insufficient space here for detailed discussion, Weiler et al. [40, 41] present equations and examples for estimating mass-loss rates, and changes therein, from presupernova systems, evidence for binarity in presupernova systems, and evidence for ionized hydrogen along the line-of-sight, possibly from the HII region which was the birthplace of the massive supernova progenitor.

\section{SN1993J}

A particularly well studied case of a radio supernova is SN 1993J [42] where fitting of the data (see Figure 1) with standard models required splitting it into two parts: an "early" data set from day 0 through day $\sim 3100$ and a "late" data set for days $\sim 3100$ through the final measurements on day 4930 . The reason for this splitting of the data 


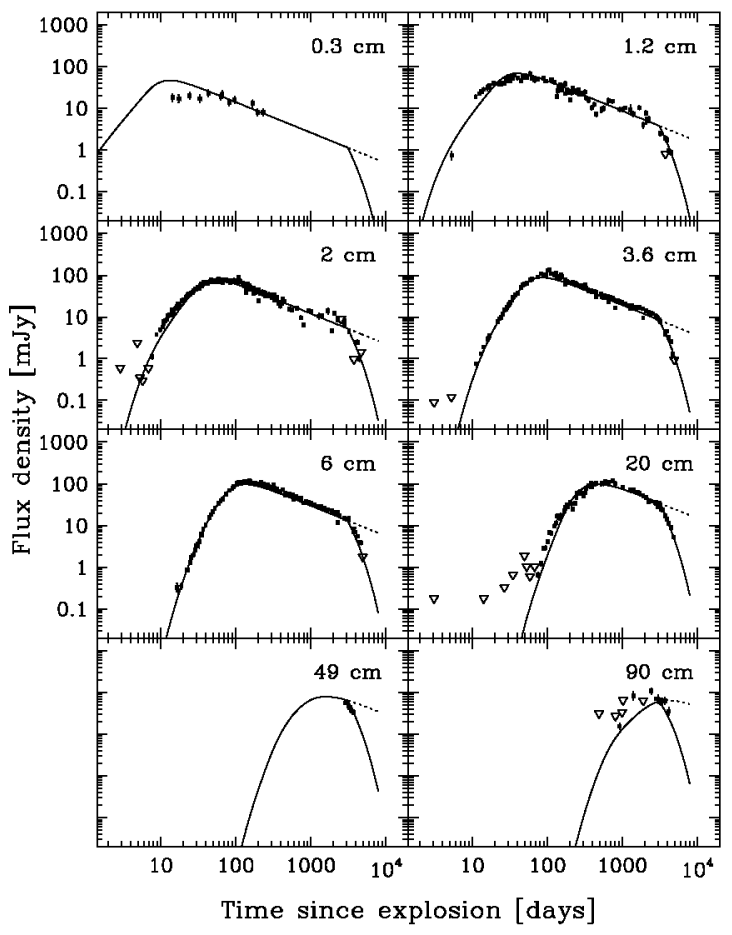

FIGURE 2. The radio light curves for SN 1993J are plotted from left to right and top to bottom at 0.3 , $1.2,2,3.6,6,20,49$, and $90 \mathrm{~cm}$. The lines represent the best fit combined synchrotron self-absorption (SSA) and thermal, free-free absorption (FFA) model as described in the text and by Weiler et al. [42] with an exponential flux density decline after day 3100 with an e-folding time of 1100 days. Upper limits $(3 \sigma)$ are shown as open inverted triangles).

set was that the decline rate $\beta$ is clearly steepening after day $\sim 3100$ and appears best described by an exponential with e-folding time of 1100 days rather than the power law decline used at earlier epochs.

The early (day < 3100 ) data were first fitted with two possible "pure" absorption models, namely pure SSA (i.e. negligible FFA at all times) and pure FFA (i.e. negligible SSA at all times). Weiler et al. [42] found that both "pure" models were able to represent the light curves as well as the spectral index evolution fairly well. However, for the pure SSA model the rising branch of the light curve at early times was "too straight" in that it could not reproduce the apparent curvature in the flux density turn-on that is most noticeable at frequencies lower than $5 \mathrm{GHz}$. Also, the spectral index evolution at early times for pure SSA was clearly inadequate to represent the observations. Moreover, the corresponding brightness temperature evolution was rather strange in that the temperature appeared to peak at later times for lower frequencies.

For the pure FFA model the rising branch of the light curves appeared to provide a better fit for both the light curves and the spectral index, but it failed the test of the brightness temperature. After correction for the external, thermal absorption, the implied 


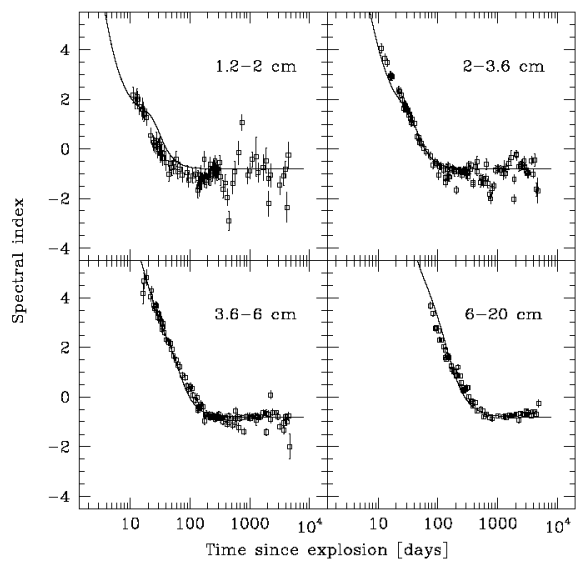

FIGURE 3. The spectral index $\left(\alpha ; \mathrm{S} \propto v^{+\alpha}\right)$ evolution for SN 1993J between 1.2 and $2 \mathrm{~cm}$ (top left), between 2 and $3.6 \mathrm{~cm}$ (top right), between 3.6 and $6 \mathrm{~cm}$ (bottom left), and between 6 and $20 \mathrm{~cm}$ (bottom right). As in Figure 2 the lines represent the best fit combined synchrotron self-absorption (SSA) and thermal, free-free absorption (FFA) model as described in the text and by Weiler et al. [42].

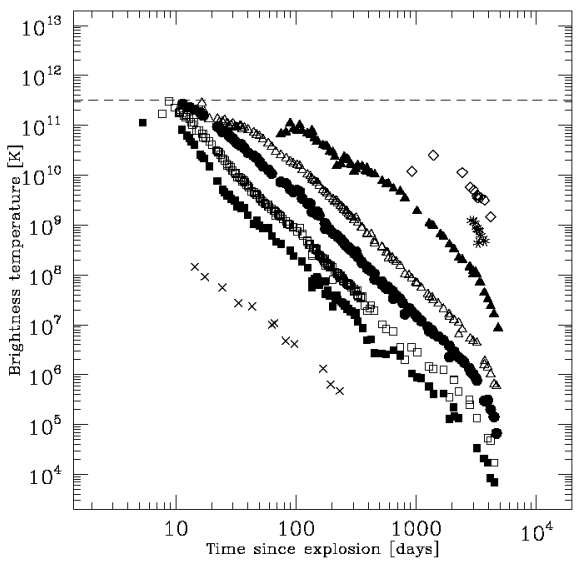

FIGURE 4. The brightness temperature $\left(\mathrm{T}_{B}\right)$ evolution for SN 1993J for, from left to right, $0.3(\times), 1.2$ (filled square), 2 (open square), 3.6 (filled circle), 6 (open triangle), 20 (filled triangle), 49 (*), and 90 (open diamond) $\mathrm{cm}$ corrected for extended, free-free absorption flux density suppression at early times as described in the text and by Weiler et al. [42]. The horizontal dashed line denotes the limiting value of $\mathrm{T}_{B} \simeq 3 \times 10^{11} \mathrm{~K}$ [27], which is not exceeded at any frequency.

brightness temperature at early times, for several frequencies, exceeded the physical limit of $T_{B} \simeq 3 \times 10^{11} \mathrm{~K}$ [see, e.g., 27].

Thus, it was clear that no "simple" model, either pure SSA or pure FFA, could account for all observational aspects of the data and a combination of the two absorption mechanisms had to be at work.

The best results were achieved with a model that comprised both SSA and FFA and 
the results are displayed in Figures 2, 3, and 4 which show the light curves, the spectral index evolution, and the brightness temperature evolution, respectively. As mentioned above, after day $\sim 3100$ an exponential with an e-folding time of 1100 days is the plotted curve to show the sharp drop-off in flux density.

\section{CONCLUSIONS}

The success of the basic parameterization and modeling is shown in the good agreement between the model fits and the data for all subtypes of RSNe. Thus, the radio emission from SNe appears to be relatively well understood in terms of blastwave interaction with a structured CSM and allows description of such progenitor system properties as massloss rate, change in mass-loss rate, filamentation or clumpiness, binarity, and remote HII, as well as estimates of progenitor properties.

We also discuss detailed radio observations of SN 1993J at multiple wavelengths for $\sim 13$ years after explosion. The radio emission for SN 1993J evolves regularly in both time and frequency, and the usual interpretation in terms of shock interaction with a complex circumstellar medium (CSM) formed by a pre-supernova stellar wind, and the inclusion of thermal and/or non-thermal absorption at early times, describes the observations rather well considering the complexity of the phenomenon. However, there are some notable characteristics peculiar to SN 1993J. 1) At a time around day

$\sim 3100$ after shock breakout the decline rate of the radio emission steepens from $\left(\mathrm{t}^{+\beta}\right)$ $\beta \sim-0.7$ to $\beta \sim-2.7$ without change in the spectral index $\left(v^{+\alpha} ; \alpha \sim-0.81\right)$. This variation, however, can better be described in terms of an exponential decay starting at day $\sim 3100$ with an e-folding time of $\sim 1100$ days. 2) The spectral index appears constant throughout our measurement era. 3) The best overall fit to all of the "early" (1.e., before day 3100 ) data is a model including both non-thermal synchrotron self-absorption (SSA) and thermal free-free absorbing (FFA) components, evolving to a constant decline rate until the break at day $\sim 3100$. In particular, neither a pure SSA nor a pure FFA absorbing model can provide a fit that simultaneously reproduces the light curves and the spectral index evolution and provides a physically realistic brightness temperature evolution.

\section{ACKNOWLEDGMENTS}

We are indebted to the VLA TAC and schedulers for permitting and arranging our numerous observations over many years and to observers who have contributed data at other radio wavelengths, sometimes unpublished. KWW wishes to thank the Office of Naval Research (ONR) for the 6.1 funding supporting his research. CJS is a Cottrell Scholar of Research Corporation and work on this project has been supported by the NASA Wisconsin Space Grant Consortium. NP is Astronomer Emeritus at the Space Telescope Science Institute (STScI) that kindly provided research facilities and partial support for this work. Additional information and data on radio supernovae can be found on http://rsd-www.nrl.navy.mil/7213/weiler/sne-home.html and linked pages. 


\section{REFERENCES}

1. Y. Andrillat, P. Prugniel, J.-M. Perelmuter, J. D. Shanklin, R. Kohl, F. Cianciolo, R. Gearhart, and A. Dosaj, IAUC, $5736(1993)$

2. N. Bartel, et al. , Nature, 368, 610 (1994)

3. N. Bartel, et al. , Astrophys. J., 581, 404 (2002)

4. M. F. Bietenholz, N. Bartel, and M. P. Rupen, Astrophys. J., 557, 770 (2001)

5. M. F. Bietenholz, N. Bartel, and M. P. Rupen, Astrophys. J., 597, 374 (2003)

6. P. Chandra, S. Bhatnagar, and A. Ray, IAUC, 7657 (2001)

7. R. A. Chevalier, Astrophys. J., 259, 302 (1982)

8. R. A. Chevalier, Astrophys. J. Letters, 259, L85 (1982)

9. R. A. Chevalier, Astrophys. J., 499, 810 (1998)

10. A. V. Filippenko, T. Matheson, R. P. Kirshner, B. P. Schmidt, N. Caldwell, J.-C. Merlin, A. Neely, P. Schmeer, and T. Vanmunster, $I A U C, 5740$ (1993)

11. A. V. Filippenko, T. Matheson, and L. Ho., Astrophys. J., 415, L103 (1993)

12. A. Filippenko, T. Matheson, and A. Barth, Astron. J., 108, 222 (1994)

13. J. M. Marcaide, et al., IAUC, 5785 (1993)

14. J. M. Marcaide, et al. , IAUC, 5820 (1993)

15. J. M. Marcaide, et al., Astrophys. I. Letters, 424, L25 (1994)

16. J. M. Marcaide, et al., Astrophys. J. Letters, 486, L31 (1997)

17. J. M. Marcaide, et al. , in press (2007)

18. M. J. Montes, K. W. Weiler, and N. Panagia, Astrophys. J., 488, 792 (1997)

19. M. J. Montes, S. D. Van Dyk, K. W. Weiler, R. A. Sramek, and N. Panagia, Astrophys. J., 506, 874 (1998)

20. M. J. Montes, K. W. Weiler, S. D. Van Dyk, N. Panagia, C. K. Lacey, R. A. Sramek, and R. Park, Astrophys. I., 532, 1124 (2000)

21. K. Nomoto, T. Suzuki, T. Shigeyama, S. Kumagai, H. Yamaoka, and H. Saio, Nature, 364, 507 (1993)

22. J. A. Phillips and S. R. Kulkarni, IAUC, 5763 (1993)

23. J. A. Phillips and S. R. Kulkarni, IAUC, 5775 (1993)

24. G. G. Pooley and D. A. Green, $I A U C, 5751$ (1993)

25. G. G. Pooley and D. A. Green, Monthly Notices Roy. Astron. Soc., 264, 17P (1993)

26. S. Radford, R. Neri, S. Guilloteau, and D. Downes, IAUC, 5768 (1993)

27. A. C. S. Readhead, Astrophys. J., 426, 51 (1994)

28. J. Ripero, F. Garcia, D. Rodriguez, IAUC, 5731 (1993)

29. J. Spyromilio, Monthly Notices Roy. Astron. Soc., 266, 61 (1994)

30. D. A. Swartz, A. Clocchiatti, R. Benjamin, D. F. Lester, and J. C. Wheeler, Nature, 365, 232 (1993)

31. R.A. Sramek, N. Panagia, and K. W. Weiler, Astrophys. J. Letters, 285, L59 (1984)

32. S. D. Van Dyk, K. W. Weiler, M. P. Rupen, R. A. Sramek, and N. Panagia, IAUC, 5759 (1993)

33. S. D. Van Dyk, H.-A. Nguyen, K. W. Weiler, R. A. Sramek, M. P. Rupen, and N. Panagia, IAUC, $5828(1993)$

34. S. D. Van Dyk, K. W. Weiler, R. A. Sramek, M. P. Rupen, and N. Panagia, Astrophys. J. Letters, 432, L115 (1994)

35. K. W. Weiler, N. Panagia, and R. A. Sramek, Astrophys. J., 364, 611 (1990)

36. K. W. Weiler, S. D. Van Dyk, N. Panagia, R. A. Sramek, and J. Discenna, Astrophys. J., 380, 161 (1991)

37. K. W. Weiler, S. D. Van Dyk, J. Pringle, and N. Panagia, Astrophys. J., 399, 672 (1992)

38. K. W. Weiler, S. D. Van Dyk, N. Panagia, and R. A. Sramek, Astrophys. J., 398, 248 (1992)

39. K. W. Weiler, R. A. Sramek, S. D. Van Dyk, and N. Panagia, LAUC, 5752 (1993)

40. K. W. Weiler, N. Panagia, M. J. Montes, and R. A. Sramek, Ann. Rev. Astron. and Astrophys., 40, $387(2002)$

41. K. W. Weiler, N. Panagia, and M. J. Montes, in "Supernovae and Gamma-Ray Bursters," K. W. Weiler, ed., Springer-Verlag, Berlin, p. 367 (2003)

42. K. W. Weiler, C. L. Williams, N. Panagia, C. J. Stockdale, M. T. Kelley, R. A. Sramek, S. D. Van Dyk, and J. M. Marcaide, Astrophys. J., in press (2007)

43. C. L. Williams, N. Panagia, C. K. Lacey, K. W. Weiler, R. A. Sramek, and S. D. Van Dyk, Astrophys. J., 581, 396 (2002) 